\title{
Morphological properties of airbrush spray-deposited enzymatic cellulose thin films
}

\author{
Wiebke Ohm, André Rothkirch, Pallavi Pandit, Volker Körstgens, Peter Müller-Buschbaum, Ramiro Rojas, \\ Shun Yu, Calvin J. Brett, Daniel L. Söderberg, Stephan V. Roth
}

(C) The Author(s) 2018

\begin{abstract}
We investigate the layer formation of enzymatic cellulose by airbrush spray coating on silicon oxide surfaces. The layer structure and morphology of enzymatic cellulose films in the thickness range between $86 \mathrm{~nm}$ and $2.1 \mu \mathrm{m}$ is determined as a function of the spray coating procedures. For each spray coating step, layer buildup, surface topography, crystallinity as well as the nanoscale structure are probed with atomic force microscopy and surfacesensitive X-ray scattering methods. Without intermittent drying, the film thickness saturates; with intermittent drying, a linear increase in layer thickness with the number of spray pulses is observed. A closed cellulose layer was always observed. The crystallinity remains unchanged; the nanoscale structures show three distinct sizes. Our results indicate that the smallest building blocks increasingly contribute to the morphology inside the cellulose
\end{abstract}

This paper was presented at the 13th Coatings Science International Conference (COSI) on June 26-30, 2017, in Noordwijk, The Netherlands.

Electronic supplementary material The online version of this article (https://doi.org/10.1007/s11998-018-0089-9) contains supplementary material, which is available to authorized users.

W. Ohm ( $₫)$, A. Rothkirch, P. Pandit,

C. J. Brett, S. V. Roth $(\bowtie)$

Deutsches Elektronen-Synchrotron (DESY), Notkestr. 85,

22607 Hamburg, Germany

e-mail: wiebke.ohm@desy.de

S. V. Roth

e-mail: stephan.roth@desy.de; svroth@kth.se

V. Körstgens, P. Müller-Buschbaum

Lehrstuhl für Funktionelle Materialien, Physik-Department,

Technische Universität München, James-Franck-Str. 1,

85748 Garching, Germany network for thicker films, showing the importance of tailoring the cellulose nanofibrils. For a layer-bylayer coating, intermittent drying is mandatory. Graphical Abstract

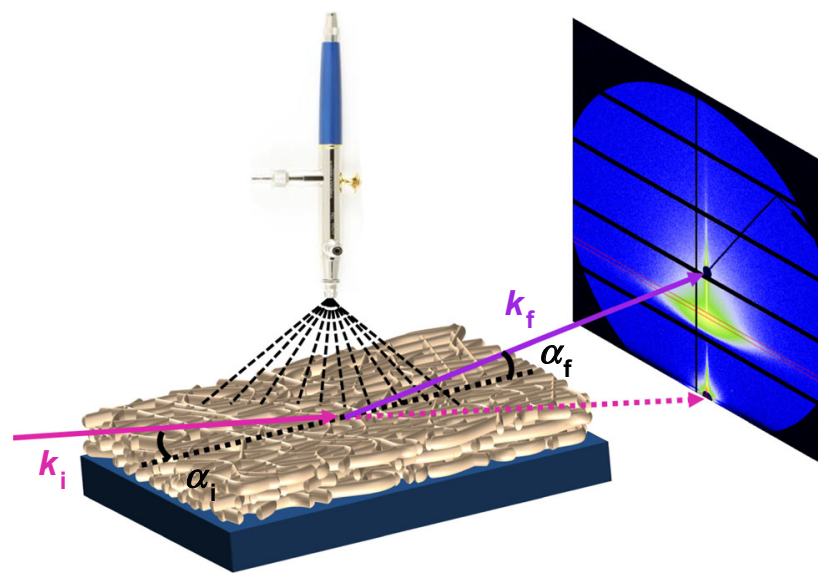

Keywords Cellulose, Thin films, Grazing incidence $\mathrm{X}$-ray scattering, Spray coating

R. Rojas, S. Yu, S. V. Roth

Department of Fibre and Polymer Technology, KTH Royal Institute of Technology, Teknikringen 56-58,

10044 Stockholm, Sweden

R. Rojas, S. Yu, C. J. Brett, D. L. Söderberg

Wallenberg Wood Science Center, KTH Royal Institute of Technology, Teknikringen 56-58, 10044 Stockholm, Sweden

C. J. Brett, D. L. Söderberg

Department of Mechanics, KTH Royal Institute of

Technology, Osquars Backe 18, 10044 Stockholm, Sweden 


\section{Introduction}

Cellulose-based films are excellent templates and coatings for bio-based applications due to their renewable and resource-saving characteristics. ${ }^{1}$ This is owing to the fact that nanoscale subunits in terms of cellulose nanocrystals and cellulose nanofibrils can be extracted and used to form ultrastrong fibrillar materials. ${ }^{2-4}$ Functionalization of cellulose films leads to flame-retardant, barrier as well as transparent devices based on cellulose. ${ }^{5-10}$ Cellulose layers can further be modified by spray coating of colloidal suspension to create superhydrophobic, yet transparent paper. ${ }^{11}$ The quest to create conductive paper ${ }^{12}$ can be achieved, for example, by incorporating carbon nanotubes. ${ }^{13}$ Furthermore, by incorporating metal nanoparticles, three-dimensional surface-enhanced Raman scattering sensors were demonstrated. ${ }^{14,15}$ Cellulose serves as scaffold material, ${ }^{16}$ barrier material, ${ }^{5}$ a template for polyelectrolyte layers, ${ }^{17}$ and even organic light-emitting diodes (OLEDs) can be installed on cellulose templates. ${ }^{18}$ Furthermore, cellulose films can be used as membranes. ${ }^{19}$ Functionalization via tin-doped indium oxide, carbon nanotubes and silver nanowires allows for creating transparent and/or conductive paper. ${ }^{13,20}$ Crucial for the aforementioned applications is the ability to fabricate coatings on a large scale and in a rapid way. Methods of coating cellulose include spincoating, dip-coating and spraying. ${ }^{17,21,22}$ Cellulose substrates show a porous structure and thus themselves might be further functionalized via spray coating. ${ }^{23}$ Spray coating itself is one method for layer-by-layer assembly in multilayered systems. ${ }^{24}$ It has recently been applied to fabricating cellulose thin films. $^{22,25}$ Such a layer-by-layer method offers the possibility of scalable and conformal fabrication and enables developments of hybrid materials. ${ }^{26}$ In particular, spray coating methods allow for an easy incorporation into roll-to-roll (R2R) application. ${ }^{27}$ It is not limited to a particular cellulose type. In addition to plant-based cellulose, there exist nonplant sources, e.g., bacterial cellulose, ${ }^{28,29}$ which could also be deposited via spray coating.

This paper is structured as follows. First, the methods and procedures for data analysis used, namely surface-sensitive X-ray scattering and atomic force microscopy, as well as the procedures for analysis of grazing incidence data, are presented. The next section describes in detail the sample preparation, i.e., the cellulose nanofibrils isolation and the enzymatic cellulose thin film preparation. Afterward, the results from the topographical studies, the crystallinity, and the nanoscale structure and morphology are discussed. The paper concludes with a summary.

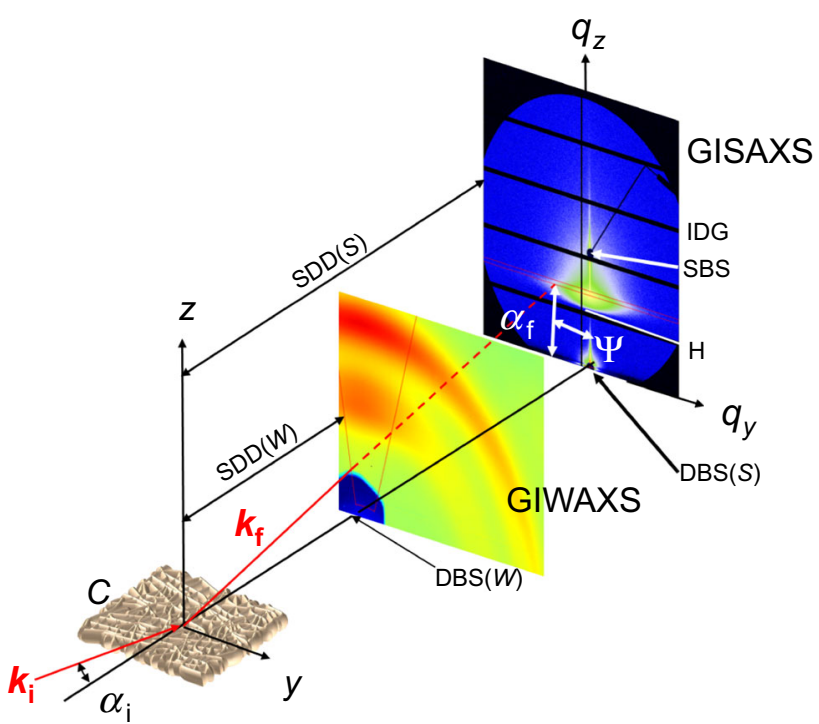

Fig. 1: Schematic setup of the grazing incidence smalland wide-angle X-ray scattering (GIWAXS/GISAXS) geometry. While $y / z$ denotes a real-space coordinate system, $q_{y}, q_{z}$ denotes a reciprocal space coordinate system. The incident $X$-ray beam is characterized by the wavevector $k_{i}=2 \pi / \lambda$, with $\lambda$ being the wavelength. $C$ denotes the cellulose sample. The scattered wavevector is denoted as $k_{\mathrm{f}} \cdot \alpha_{\mathrm{i}}, \alpha_{\mathrm{f}}, Y$ denote the angle of incidence, the exit angle, and out-of-plane angle (with respect to the scattering plane spanned by $z$ and $\left.k_{i}\right) \cdot \operatorname{SDD}(W, S)$ denote the distance of the sample to the detector for the GIWAXS and GISAXS case. IDG denotes the intermodule gap of the two-dimensional Pilatus detector. $\operatorname{DBS}(W, S)$ and SBS denote the beamstop for the direct beam for GIWAXS and GISAXS and the specular beamstop in GISAXS geometry. $H$ denotes the sample horizon

\section{Methods}

\section{Surface-sensitive X-ray scattering}

X-ray diffraction and scattering are chosen to investigate the structure and morphology of cellulose thin films and fibers. ${ }^{21,30-35}$ In grazing incidence scattering, the X-ray beam impinges under a low angle, typically $\alpha_{i}<1^{\circ}$ onto the sample surface, ${ }^{36-39}$ see Fig. 1. Grazing incidence small-angle X-ray scattering (GISAXS) was first introduced by Levine and coworkers. ${ }^{40}$ The sample is mounted on a remotely controlled goniometer, allowing for adjusting the incident angle as well as the surface inclination perpendicular to the incoming beam. Additionally, the sample can be translated in $y$ direction and $z$-direction. The X-ray beam is characterized by the impinging wavevector $\vec{k}_{\mathrm{i}}$ with an absolute value $\left|\vec{k}_{\mathrm{i}}\right|=\frac{2 \pi}{\lambda}$ and $\lambda$ being the wavelength. 
The scattering wavevector $\vec{k}_{\mathrm{f}}$ with an absolute value $\left|\vec{k}_{\mathrm{f}}\right|=\frac{2 \pi}{\lambda}$ in the case of elastic scattering is considered here. The wavevector transfer is defined using the following equation

$\vec{q}=\vec{k}_{\mathrm{f}}-\overrightarrow{k_{\mathrm{i}}}$

The three components of $\vec{q}$ can be written in GISAXS geometry as

$$
\begin{aligned}
& q_{x}=\frac{2 \pi}{\lambda}\left(\cos \left(\alpha_{\mathrm{f}}\right) \cos (\Psi)-\cos \left(\alpha_{\mathrm{i}}\right)\right) \\
& q_{y}=\frac{2 \pi}{\lambda}\left(\cos \left(\alpha_{\mathrm{f}}\right) \sin (\Psi)\right) \\
& q_{z}=\frac{2 \pi}{\lambda}\left(\sin \left(\alpha_{\mathrm{i}}\right)+\sin \left(\alpha_{\mathrm{f}}\right)\right)
\end{aligned}
$$

Typically, $q_{x} \ll q_{y}, q_{z}$ and can thus be neglected.

The incident wave is scattered at electron density differences in the thin film. At $\alpha_{\mathrm{f}}=\alpha_{\mathrm{i}}$, the X-ray beam is specularly reflected following the simple laws of optics. In our case, this specular reflected beam is shadowed by a lead beamstop in order to avoid saturation of the detector. The diffusely scattered intensity is detected outside this area above the sample horizon $(H)$. The scattered intensity is determined by reflection and refraction effects and the roughness of the film. The scattered intensity consists of a convolution of structure factors and form factors of the structures present inside the thin film. The structure factor $S\left(q_{y}, q_{z}\right)$ is determined by the spatial arrangement of the scattering centers, while the form factor $F\left(q_{y}, q_{z}\right)$ is by their shape, i.e., radius and height. The form factor is related to the electron density distribution of the thin film via:

$F\left(q_{y}, q_{z}\right)=\int_{V} \rho(\vec{r}) \mathrm{e}^{-i \vec{q} \vec{r}} d^{3} r$

An interference maximum between transmitted beam and incoming beam occurs when the incident or exit angle corresponds to the angle of total reflection $\alpha_{i, f}=\alpha_{c}$. The critical angle can be calculated as $\alpha_{c}=$ $\sqrt{2 \delta} \propto \sqrt{\rho}$ where the dispersion $\delta$ is related to the complex index of refraction via $n=1-\delta+i \beta . \quad \beta$ denotes the absorption and $\rho$ the density of the material. This interference maximum is called Yoneda peak $^{41}$ and can be calculated as a maximum in the Fresnel transmission coefficients. ${ }^{42}$ It should be noted that GISAXS offers the opportunity for analyzing buried structures ${ }^{43,44}$ as well as complex nanostructures. ${ }^{45,46}$

\section{Atomic force microscopy}

For characterization of surface morphology and probing film thicknesses, atomic force microscopy (AFM), using an NT-MDT NTEGRA Aura system equipped with HA_NC tips (tip curvature radius $<10 \mathrm{~nm}$ ), was applied in tapping mode under ambient conditions. A scratch was done at the center of samples, and three AFM images for each sample were recorded at the scratch position. The height difference from substrate to layer surface was measured at $10-15$ positions for each AFM image. The mean value of these height measurements is taken as the film thickness with the corresponding standard deviation. Root-mean-square (rms) roughness of the cellulose films was calculated from the surface height profile of 3 AFM images at different spots of the samples with an image size of $15 \mu \mathrm{m} \times 15 \mu \mathrm{m}$.

\section{Procedures for analysis of grazing incidence data}

The grazing incidence small-angle X-ray scattering (GISAXS) as well as the corresponding wide-angle scattering (GIWAXS) signal of cellulose films was recorded to gain information about the inner film morphology and crystallinity. The measurements were taken at beamline $\mathrm{P} 3^{47}$ of PETRA III storage ring at DESY (Hamburg, Germany). The X-ray beam had a wavelength $\lambda=0.97 \AA$ and a beam size of $22 \mu \mathrm{m} \times 16$ $\mu \mathrm{m}$ (horizontal $\times$ vertical). A two-dimensional (2D) scattering pattern was recorded using a Pilatus $1 \mathrm{M}$ detector $(981 \times 1043$ pixel, pixel size $172 \mu \mathrm{m} \times 172$ $\mu \mathrm{m})$ in GISAXS and Pilatus $300 \mathrm{k}$ detector $(487 \times 619$ pixel, pixel size $172 \mu \mathrm{m} \times 172 \mu \mathrm{m})$ in GIWAXS geometry. The sample-to-detector distance (SDD) for GISAXS and GIWAXS was determined to be $\mathrm{SDD}_{\text {GISAXS }}=4976 \mathrm{~mm} \pm 2 \mathrm{~mm}$ and $\mathrm{SDD}_{\text {GIWAXs }}=$ $139 \mathrm{~mm} \pm 0.2 \mathrm{~mm}$, respectively, using silver behenate $\left(\mathrm{AgC}_{22} \mathrm{H}_{43} \mathrm{O}_{2}\right)$, Lanthanhexaborid (LaB6) standard and the d-spacing of cellulose according to French. ${ }^{48}$ The angle of incidence $\left(\alpha_{i}\right)$ was set to $\alpha_{i}=0.42^{\circ} \pm 0.01^{\circ}$. This is well above the critical angle $\left(\alpha_{c}\right)$ of cellulose with $\alpha_{c \text {,cellulose }}=0.117^{\circ}$ leading on one hand to clear separation of the Yoneda ${ }^{41}$ region from the specular reflected beam that is covered with a beamstop (SBS in Figs. 1, 4a). On the other hand, the high angle of incidence allows for probing thick layers, since the scattering depth at the Yoneda region of cellulose is already in the micrometer range for this material system.

The square samples were placed with two edges parallel to the X-ray and were aligned close to the edges of the substrate to avoid unnecessary beam exposure during alignment at the measurement area as much as possible. Half of the substrate $(\sim 7 \mathrm{~mm} \times 20$ $\mathrm{mm}$ ) was then scanned in GISAXS geometry and the other half in GIWAXS geometry with a step size of $0.1 \mathrm{~mm}$ by translating the sample through the beam in $y$-direction (see Fig. 1). The single-image exposure was 
$0.1 \mathrm{~s}$ for GISAXS and $0.5 \mathrm{~s}$ for GIWAXS, respectively. Preliminary GISAXS measurements at one position outside the scanned areas were recorded to evaluate beam damage of cellulose layers by acquiring 100 images of nominal 0.1-s exposure time. To assess the beam damage, the images taken after different total exposure were compared to each other. These beam damage measurements demonstrated that the cellulose layers can withstand an exposure of $2 \mathrm{~s}$ at the applied beam intensity and energy. Thus, we assure that the chosen exposure time did not affect the samples. To increase statistics while maintaining the exposure below the beam damage limit, we summed up 30 images for further analysis. Data reduction was performed by extracting one-dimensional intensity distributions $I\left(q_{y}\right)$ and $I\left(q_{z}\right)$ using the software DPDAK. ${ }^{49}$

\section{Sample preparation}

\section{Cellulose nanofibril preparation}

Enzymatic cellulose was prepared following Henriksson et al. ${ }^{50}$ Never-dried spruce sulfite pulp (Nordic Paper Säffle, Sweden) was treated with the enzyme mono-component cellulase FiberCare R (Novozymes, Denmark). For the nanofibrillation, the enzymetreated pulp was processed on an M-110EH Microfluidizer (Microfluidics, USA) at 800 bar through a chamber system of 400 and $200 \mu \mathrm{m}$ in diameter for 3 cycles, followed by 1800 bar through a chamber system of 200 and $100 \mu \mathrm{m}$ in diameter for 5 cycles. Concentration was determined to be $15 \pm 2 \mathrm{~g} / \mathrm{L}$ by weighing of a droplet before and after drying.

\section{Thin film preparation}

The polished silicon wafer substrates (Si-Mat, $20 \mathrm{~mm} \times 20 \mathrm{~mm}$ ) were cleaned with acetone in ultrasonic bath for $10 \mathrm{~min}$ prior to rinsing with acetone, isopropanol, methanol and deionized water. The precleaned substrates were kept in acid bath $\left[60 \mathrm{~mL} \mathrm{H}_{2} \mathrm{O}\right.$, $140 \mathrm{~mL} \mathrm{H}_{2} \mathrm{O}_{2}$ (concentration $30 \%$ ) and $304 \mathrm{~mL} \mathrm{H}_{2} \mathrm{SO}_{4}$ (concentration $96 \%$ )] at $80^{\circ} \mathrm{C}$ for $15 \mathrm{~min}$. Later, they were rinsed with deionized water to remove residual acid and stored in a water bath until further processing. This cleaning procedure leads to a hydrophilic surface. ${ }^{51}$ Right before coating with cellulose, the substrates were dried with compressed $\mathrm{N}_{2}$. As precursor solution, we used enzymatic cellulose nanofibrils dispersed in water with a concentration of $15 \pm 2 \mathrm{~g} / \mathrm{L}$ $(1.2 \mathrm{wt} \%)$. The initial dispersion was further diluted in $\mathrm{H}_{2} \mathrm{O}$ to a final concentration of $0.3 \mathrm{wt} \%$ cellulose in water. Due to the nanometer size of the cellulose fibrils, solutions exceeding concentrations of $0.5 \mathrm{wt} \%$ show highly viscous behavior and are not suitable for spraying as they might block the spray nozzle of the airbrush system used. Hence, we chose a concentration of $0.3 \mathrm{wt} \%$ cellulose in water to balance between wearout of spray device and efficient material deposition suitable for industrial application. For spraying of cellulose thin films, a commercial airbrush spray nozzle (Harder \& Steenbeck, model Grafo T3) was installed above the sample position. ${ }^{52-57}$ The sample-to-nozzle distance (SND) was set to $15 \mathrm{~cm}$. We used an $\mathrm{N}_{2}$ pressure of $p=1 \mathrm{bar}$ and a spray rate of $(0.10 \pm 0.01) \mathrm{mL} / \mathrm{s}$. The sample was kept at room temperature. We performed short spray pulses of $0.4 \mathrm{~s}$ with breaks of $0.1 \mathrm{~s}$. The effect of increasing number of spray pulses on the morphology of cellulose thin films is the focus of this work. We compared samples of different spray protocols: $1,2,4,8,10,12,15,20,30,50$ and 100 pulses performed with breaks (interposition time) of $0.1 \mathrm{~s}$ which is not sufficient for drying of the film. In comparison, samples with 20, 50 and 60 pulses were prepared with $\sim 30$-min breaks after each 10 pulses; these breaks allowed for complete drying of the film.

\section{Results and discussion}

\section{Topographical information}

The AFM images show a dense cellulose nanofibril network for all sprayed samples, as demonstrated in Figs. 2a-2c for samples prepared with 1,8 and 30 spray pulses. Individual fibrils can be identified in all images. For spraying at normal incidence, no preferential orientation is expected which is in agreement with the experimental results. Already for a spray time of $0.4 \mathrm{~s}$ (single spray pulse), the cellulose network is fully closed. In Fig. 2d, the film thickness is displayed as a function of spray pulses. The thickness increases linearly up to a layer thickness of $\sim 1200 \mathrm{~nm}$, corresponding to 30 spray pulses. For a higher number of spray pulses, we observe a slight decrease in film thickness though more material is deposited. For this unexpected behavior, two reasons are possible: (1) compaction of cellulose film due to higher material deposition and (2) loss of solution sprayed onto the sample due to liquid shear for high amounts of solution and thus thick liquid films. From the AFM measurements, one cannot identify whether (1) or (2) is more likely; however, during the deposition a radial shearing of the liquid was visually observed giving evidence for the second reason. With interposition of drying time, e.g., in this study after each tenth spray pulse (gray symbols in Fig. 2d), this decrease in film thickness is impeded such that higher cellulose film thicknesses can be fabricated up to $\sim 2.5 \mu \mathrm{m}$ for the analyzed spray protocol. The root-mean-square (rms) roughness of the sprayed cellulose films is presented in Fig. 2e against the film thickness. We observe an increase in the rms roughness with increasing cellulose thickness for thin 
layers-up to a critical thickness of $\sim 800 \mathrm{~nm}$. For thicker cellulose layers the surface roughness is constant at values around $50 \mathrm{~nm}$. Since the thinnest cellulose layer (below $100 \mathrm{~nm}$ ) already exhibits an rms roughness of $(21 \pm 2) \mathrm{nm}$, we conclude that the cellulose surface roughness is uncorrelated with the underlying substrate roughness which is below $2 \mathrm{~nm}$ for the polished $\mathrm{Si}$ substrate. This fact is especially interesting for certain industrial applications such that sprayed cellulose films can be deposited onto various substrates maintaining their surface characteristics, to a certain extent, independent of substrate morphology.

\section{Crystallinity}

To analyze the crystallinity of cellulose nanofibril layers, we performed GIWAXS measurements. A typical two-dimensional (2D) GIWAXS pattern of a cellulose film is displayed in Fig. 3a. The transforma-
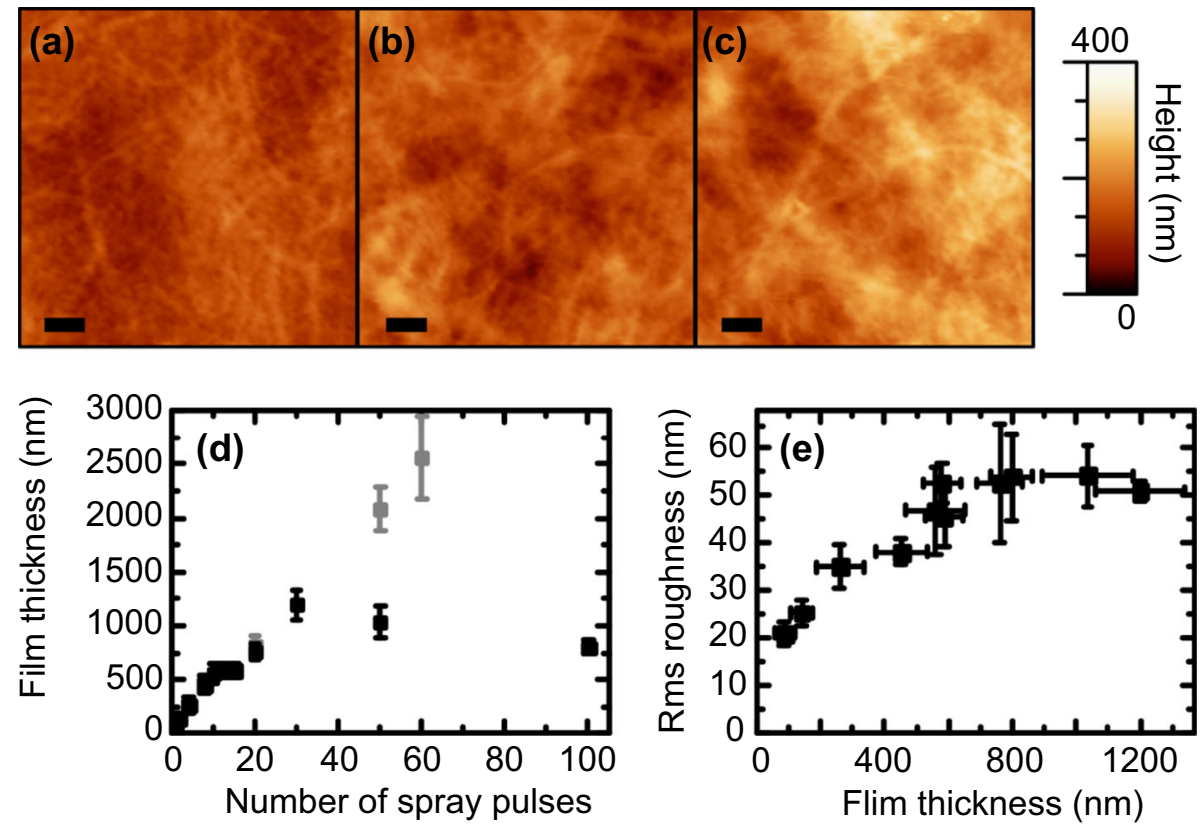

Fig. 2: (a-c) AFM images for cellulose thin films with different thicknesses: (a) $86 \mathrm{~nm} \pm 31 \mathrm{~nm}$; (b) $452 \mathrm{~nm} \pm 80 \mathrm{~nm}$; (c) $1201 \mathrm{~nm} \pm 138 \mathrm{~nm}$. The scale bar in (a-c) corresponds to $1 \mu \mathrm{m}$. (d) Cellulose film thickness displayed against the number of spay pulses (0.4-s spraying, 0.1 -s break) for $0.3 \mathrm{wt} \%$ aqueous cellulose dispersion sprayed at room temperature. Continued spray pulses (black) and with drying time after each tenth spray pulse (gray) are compared. (e) Corresponding root-meansquare (rms) roughness for the cellulose films displayed in (d) (Color figure online)
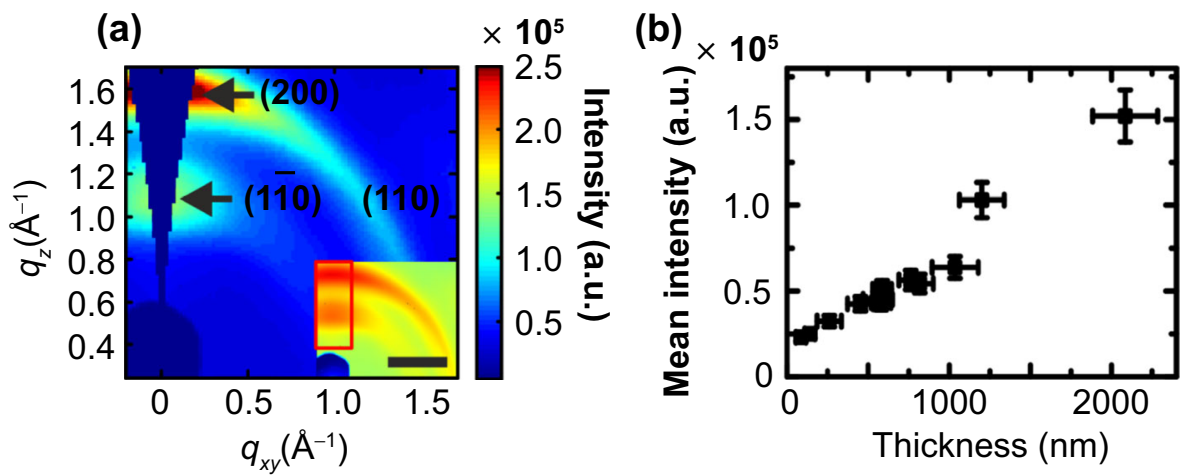

Fig. 3: (a) GIWAXS pattern of a cellulose layer on Si substrate (cellulose thickness: $1201 \mathrm{~nm}$ ) showing the main cellulose reflexes (200), (110), (110). The inset shows the 2D detector image with a scale bar (100 pixels); the color scale is logarithmic. (b) Integrated intensity of 2D detector image (integrated area marked by the red box in the inset in a) displayed against cellulose thickness. The amplitude for the thickness of $2084 \mathrm{~nm}$ was derived from the intermittent drying procedure (Color figure online) 
tion to $q_{x y}=\sqrt{q_{x}^{2}+q_{y}^{2}}$ and $q_{z}$ was performed using the software GIXSGUI. ${ }^{58}$ Please note that for GISAXS (see "Structure and morphology") typically $q_{x} \ll q_{y}$, $q_{z}$ holds and thus $q_{x y}$ reduces to $q_{y}$ in GISAXS. In the GIWAXS data, we assigned the $h k l$ values as well as the d-spacing according to the work of Nishiyama et al. ${ }^{59}$ Due to the arrangement of cellulose chains to fibrils, the (200) reflection is most pronounced in the cellulose films. Additionally, the (110) and (110) reflexes are visible. The $2 \mathrm{D}$ pattern clearly shows that sprayed cellulose layers display strong texturing, since the reflections have a strong dependence on the azimuthal angle: the intensity of the (110), (110) as well as (200) reflections is higher in the out-of-plane direction than in the in-plane direction. Hence, we may assume that the nanofibrils lay on the surface and are not standing upright, which is corroborated by the AFM images. In order to analyze the influence of the increasing cellulose film thickness on the GIWAXS signal, we plot the integrated intensity of the peaks as a function of layer thickness (see Fig. 3b). As expected, the integrated intensity increases linearly with increasing layer thickness. This is due to the fact that more material with constant crystallinity is deposited in the spray process. Neither their structure nor fibril morphology seems to change during the spray deposition process. This allows a direct monitoring of the deposited film thickness.

\section{Structure and morphology}

In Fig. 4a, a typical GISAXS pattern of a sprayed cellulose film onto an Si substrate is presented. Both the direct beam (red cross) and the specular reflected beam-denoted by SBS - are covered with a beamstop. In the cellulose scattering signal, we observe a gradual decrease in signal intensity with increasing $q_{y}$ and distance from Yoneda region of cellulose (red box). The strong scattering intensity around $q_{y}=0$ $\mathrm{nm}^{-1}$ can be mainly attributed to scattering from the polished Si substrate and mesoscale, unresolved structures (see also Fig. 4b). To further analyze the GISAXS data, we performed linecut integrations along the horizontal (red) and vertical (green) direction, as indicated in Fig. 4a. The cuts for different cellulose layer thicknesses are shown in Figs. $4 \mathrm{~b}$ and $4 \mathrm{c}$. Both graphs demonstrate an increase in scattering intensity with larger layer thickness which is more pronounced for high $q$-values. The higher scattering intensity for larger $q$-values indicates the existence of an increasing number of small structures, both in lateral and horizontal direction, leading to scattering to high $q$-values.

The nominal critical angle of cellulose is $\alpha_{\mathrm{c} \text {,cellulose }}=0.117^{\circ}$, the one of Si is $\alpha_{\mathrm{c}, \mathrm{Si}}=0.140^{\circ}$, and the one of $\mathrm{SiO}_{2}$ is $\alpha_{\mathrm{c}, \mathrm{SiO} 2}=0.135^{\circ}$. The Yoneda peak positions are marked in Fig. S2.

The sponge-like structure mentioned in reference (60) can be interpreted as a closed network. Such a (a)

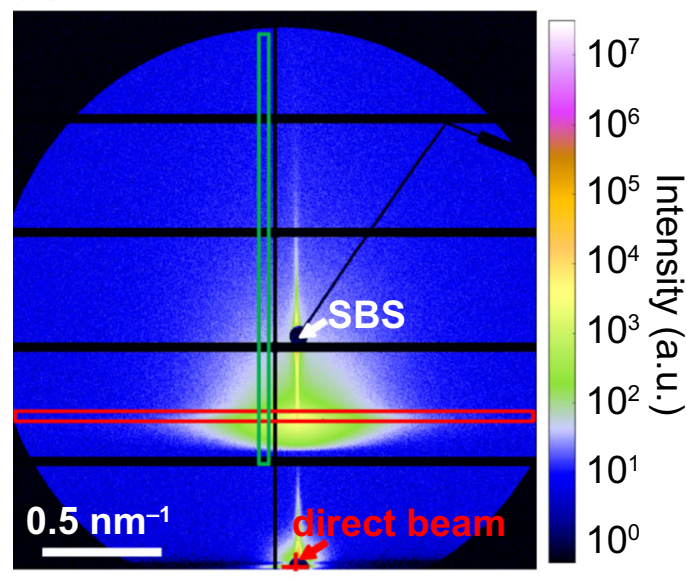

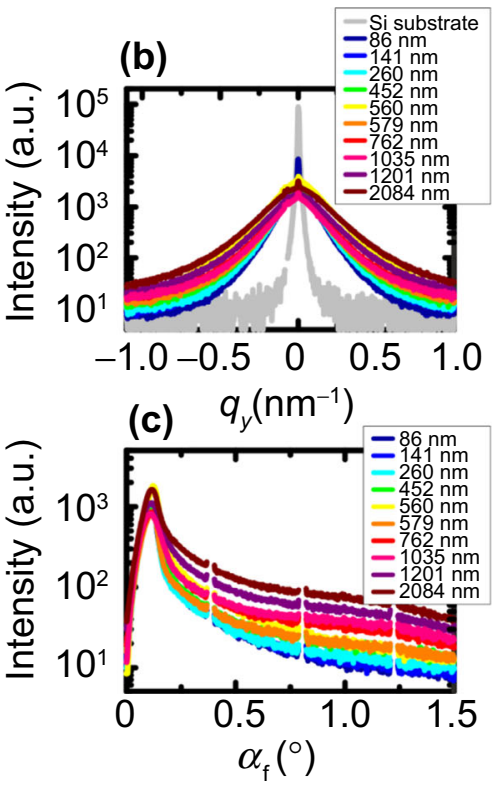

Fig. 4: (a) Characteristic two-dimensional (2D) detector image of GISAXS scattering signal from cellulose film on Si with cellulose thickness of $86 \mathrm{~nm}$. Displayed is the direct beam position (red cross) as well as the specular beamstop (SBS). The red and green box represent the areas which were integrated for extracting the horizontal (red) and vertical (green) linecuts. Horizontal (b) and vertical (c) linecuts are shown for different cellulose layer thicknesses. The corresponding cellulose thickness for the line color is denoted in the legend. The linecuts for the thickness of $2084 \mathrm{~nm}$ were derived from the intermittent drying procedure. The beamstop of the direct beam has a small photodiode incorporated to monitor the beam intensity (Color figure online) 
network or sponge-like structure is well known from photovoltaics, and we apply this concept for the first time to CNF films, as outlined now. Fitting of the Yoneda peak position from the vertical cuts using a Gaussian function (see supplementary information) resulted in values for the critical angle between $\alpha_{c}=0.110^{\circ} \pm 0.001^{\circ}$ and $\alpha_{c}=0.126^{\circ} \pm 0.001^{\circ}$ which is, within a $10 \%$ deviation, in good agreement with the theoretical value for cellulose. This behavior is expected for a closed network ${ }^{60}$ which corresponds to the findings from the AFM measurement. This provides further evidence that the thin CNF film can be considered as a closed network with rather uncorrelated surface topology compared to the substrate. Therefore, we cannot make a conclusion on the porosity from these findings.

For further quantification of the structure sizes and relative contributions, we performed fitting of the horizontal cuts applying a cylindrical model as described elsewhere. ${ }^{61}$ In short, the scattering intensity for a single structure is considered as a convolution of form factor $F(q)$ of an upright standing cylinder in Born approximation (BA) and a structure factor $S(q)$ for paracrystalline domains ${ }^{62}$ at constant $q_{z}{ }^{63}: I\left(q_{y}\right) \propto$ $S\left(q_{y}, D\right) \times\left|F\left(q_{y}, R\right)\right|^{2}$. The cylinder radius $R$ as well as the cylinder distance $D$ were subjected to the fitting procedure. Furthermore, size distribution and disorder was accounted for by introducing normal/Gaussian probability distribution of radius and next-neighbor distance. The final model consisted of the summed scattering intensity $I_{j}(j=1,2,3)$ of three different structures, with radii $R_{1}, R_{2}, R_{3}$ and distances $D_{1}, D_{2}$, $D_{3}$, respectively. To account for finite resolution of experimental measurements, ${ }^{47}$ a Gaussian shaped resolution function was added, contributing mainly to the intensity distribution at low $q_{y}<0.05 \mathrm{~nm}^{-1}$.

In Figs. 5a and $5 b$ the experimental linecuts (see Fig. 4b) are shown: (a) for a thin $(86 \mathrm{~nm} \pm 31 \mathrm{~nm})$ and (b) for a thick $(1201 \mathrm{~nm} \pm 138 \mathrm{~nm})$ cellulose layer together with the modeled intensity and the contribution of the three structures (black, blue, and red lines) as well as the resolution peak (violet). The form factor is displayed as the solid line, and the structure factor as the dashed line. Comparing the fit results for different cellulose layer thicknesses, we observe, within the error range, constant structure sizes $\left(R_{1}, R_{2}\right.$ and $\left.R_{3}\right)$ and distances $\left(D_{1}, D_{2}\right.$ and $\left.D_{3}\right)$ as summarized in Table 1 (for more details see SI). Especially for the largest structure size, the structure factor is constant in the region where the form factor declines. This follows for well-separated objects, having a much larger nextneighbor distance than the object diameter. This
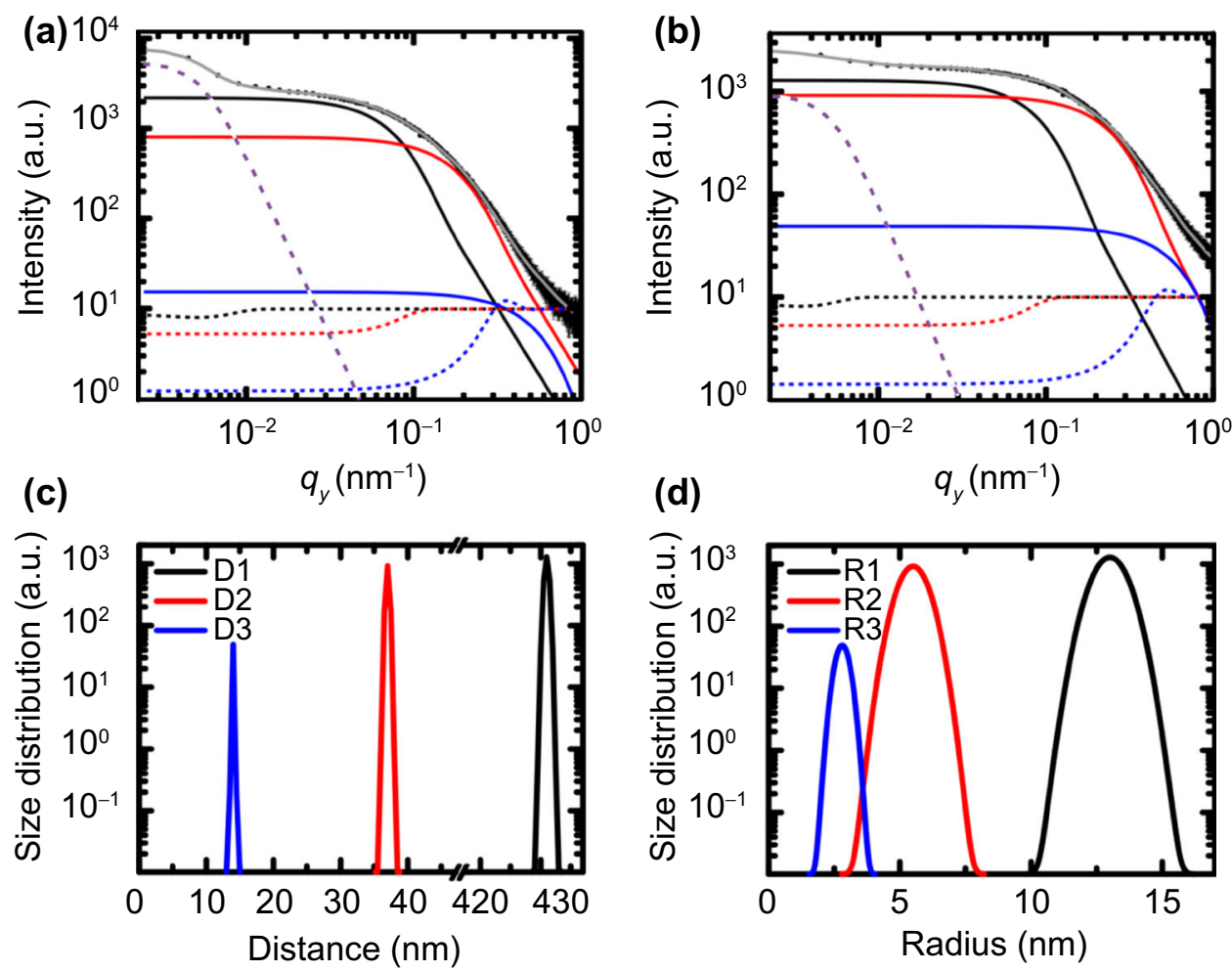

Fig. 5: Horizontal linecuts with corresponding fit using cylinder model for (a) 86-nm-thick and (b) 1201-nm-thick cellulose layer. Displayed are the form factor (solid line) and structure factor (dashed line) for a large (black), intermediate (red), and small structure (blue). The dashed violet line represents the resolution peak. The resulting curve (gray) is compared to the measured data (black dots). Corresponding distances and radii are shown in (c) and (d), respectively. Here, the amplitude of the Gaussian peaks corresponds to the statistical weight of the three structures, exemplarily illustrated for a 86-nm-thick cellulose layer (Color figure online) 
Table 1: Summarized structure sizes and distances for cylindrical model

\begin{tabular}{lccc} 
& $\begin{array}{c}\text { Structure 1 } \\
\text { Value }(\mathrm{nm})\end{array}$ & $\begin{array}{c}\text { Structure 2 } \\
\text { Value }(\mathrm{nm})\end{array}$ & $\begin{array}{c}\text { Structure 3 } \\
\text { Value }(\mathrm{nm})\end{array}$ \\
\hline Radius & $13 \pm 1$ & $5.5 \pm 1.0$ & $2.8 \pm 0.5$ \\
Distance & $431 \pm 63$ & $37 \pm 3$ & $14 \pm 2$ \\
\hline
\end{tabular}

Presented are the radii $R$ and distances $D$ with distributions for the three structures, fitted to horizontal linecuts of GISAXS pattern from Si/cellulose layers (see Fig. 4)

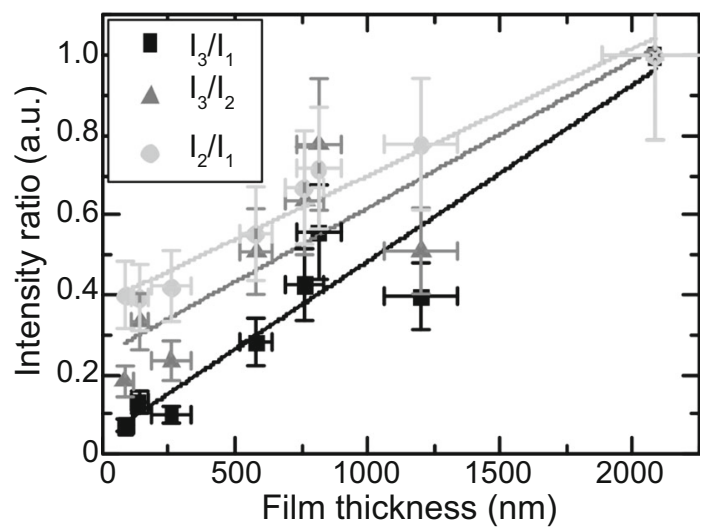

Fig. 6: Intensity ratios of the three cylinder structures from cylindrical model fit (see Fig. 5) displayed as a function of cellulose layer thickness. Depicted is the intensity ratio from structure 3 to structure 1 (black boxes), structure 3 to structure 2 (gray triangles), and structure 2 to structure 1 (light gray circles) together with a linear fit representing the increase in relative scattering contribution of small structures

behavior is less pronounced for the intermediate structure. For the smallest structure, the form factor and structure factor are affecting each other in terms of developing the scattering intensity, which is expected for close-packed objects. ${ }^{64}$ The resulting structure size distribution is summarized in Figs. 5c and 5d showing the structures' distances (c) as well as radii (d). Here, the size distribution intensities correspond to the intensities found for the $86 \mathrm{~nm} \pm 31 \mathrm{~nm}$ cellulose layer presented in Fig. 5a. However, we found the same structure sizes and distances in all the cellulose samples; the relative intensity contribution of different structure sizes changes with the cellulose thickness, as the comparison of the horizontal linecuts for different cellulose layer thicknesses (see Fig. 4b) suggests. For quantification, we examined the relative intensity of the different structures, which is presented in Fig. 6. The diagram summarizes the normalized relative intensity of small structure to large structure: $I_{3} / I_{1}, I_{3} /$ $I_{2}$ and $I_{2} / I_{1}$, displayed against the cellulose thickness. All three graphs show a linear increase in relative intensity with cellulose thickness. A linear fit resulted in a slope of: $(3.2 \pm 0.3) \times 10^{-4} \mathrm{~nm}^{-1}$ for $I_{2} / I_{1}$,
$(3.7 \pm 0.8) \times 10^{-4} \mathrm{~nm}^{-1} \quad$ for $\quad I_{3} / I_{2}$ and $\quad(4.4 \pm$ $0.6) \times 10^{-4} \mathrm{~nm}^{-1}$ for $I_{3} / I_{1}$. Thus, the increase in relative intensity with cellulose layer thickness is highest for the ratio $I_{3} / I_{1}$, comparing the smallest structure intensities $I_{3}$ with the largest structure intensities $I_{1}$. Furthermore, the ratio of the intermediate structure $I_{2}$ to the largest structure $I_{1}$ resulted in the smallest slope. Hence, the relative increase in the smallest structure signal $I_{3}$ is by a factor of 1.4 higher compared to the intermediate structure signal $I_{2}$. These results demonstrate the relative increase in smaller structures with increasing cellulose thickness.

Our analysis yields three structural sizes (radii) $13 \pm 1,5.5 \pm 1.0$, and $2.8 \pm 0.5 \mathrm{~nm}$, respectively. Similar sizes have been found recently for TEMPO cellulose dispersions using transmission SAXS. ${ }^{34}$ Spray deposition in the geometry employed here does not induce a preferential ordering in the dried cellulose film as is seen in the AFM images (Figs. 2a-2c). The model of cylinders for quantitatively analyzing the GISAXS data employed thus assumes rotational symmetry of the CNFs within the footprint of the beam. ${ }^{52,61}$ Using the trimodal distribution allows for fitting the curves. The model using cylinders was only applied to the lateral cuts for extraction of lateral structure sizes. Hence, the cylinder length is not taken into account within this model. The cellulose fibrils have a length in the $\mu \mathrm{m}$ range. These sizes are included in the resolution function, and we refrain from modeling separately larger morphologies with radii $>100 \mathrm{~nm}$ which are approaching the resolution limit. A sketch for visualization of the extracted length scales is added in fig. S5 in the SI, depicting a hierarchical structure of the fibrils consisting of smaller CNFs. We interpret the two smallest radii as fibrils with two distinct diameters. An assembly of these CNFs yields a larger structure, which might eventually build up the fibrils observed in AFM.

The linear increase in the ratio $I_{j} / I_{k}(j>k)$ might be interpreted as follows: Firstly, the enzymatic cellulose dispersion contains fibrils with different diameters; secondly, the large- and medium-sized structures might consist of the observed smaller-sized structures. This arrangement of fibril into larger entities might occur during deposition. Figure 6 thus shows that with increasing thickness this agglomeration seems to be less likely. In other words, the smallest building blocks increasingly contribute to the morphology inside the cellulose network for thicker films, showing the importance of tailoring the cellulose nanofibrils.

\section{Summary}

We have investigated the layer formation of enzymatic cellulose via spray coating. For the first time, we employed a combination of different real-space and reciprocal space analysis methods: AFM yielded the surface topography, while GIWAXS and GISAXS 
allowed for correlating the deposited layer thickness with the layer nanostructure. Depending on the spray coating procedure, the layer morphology changed. Closed networks with thicknesses in the range from $86 \mathrm{~nm}$ to larger than $1 \mu \mathrm{m}$ were achieved. The layers seem to be a closed network. At the same time, a roughness of $50 \mathrm{~nm}(\mathrm{rms})$ was observed. The nanostructure consists of three sizes $(13 \pm 1,5.5 \pm 1.0$, $2.8 \pm 0.5 \mathrm{~nm}$ ) in close-packed small entities to wellseparated medium and large entities. The crystalline signal (GIWAXS) shows a linear increase, being consistent with constant deposition during each spray cycle. Our results allow for an understanding of the spray coating of fibrillated cellulose layers on smooth surfaces. For a layer-by-layer coating, interposed drying is mandatory. Future investigations will include the study of the in situ and real-time spray coating processes using time-resolved surface-sensitive X-ray scattering.

Acknowledgments C.B. and S.V.R. acknowledge funding by the DESY strategic fund "Investigation of processes for spraying and spray coating of hybrid cellulose-based nanostructures." V.K. and P.M.-B. acknowledge Bavarian State Ministry of Education, Science and Arts for funding this research work via project "Energy Valley Bavaria." S.Y. and R.R. acknowledge the financial support from the Knut and Alice Wallenberg Foundation through the Wallenberg Wood Science Center. We thank Dr. Tomas Larsson (RISE, Stockholm, Sweden) for scientific discussions. Parts of this research were conducted at light source PETRA III at DESY, a member of the Helmholtz Association (HGF).

Open Access This article is distributed under the terms of the Creative Commons Attribution 4.0 International License (http://creativecommons.org/lice nses/by/4.0/), which permits unrestricted use, distribution, and reproduction in any medium, provided you give appropriate credit to the original author(s) and the source, provide a link to the Creative Commons license, and indicate if changes were made.

\section{References}

1. Eichhorn, SJ, Dufresne, A, Aranguren, M, Marcovich, NE, Capadona, JR, Rowan, SJ, Weder, C, Thielemans, W, Roman, M, Renneckar, S, Gindl, W, Veigel, S, Keckes, J, Yano, H, Abe, K, Nogi, M, Nakagaito, AN, Mangalam, A, Simonsen, J, Benight, AS, Bismarck, A, Berglund, LA, Peijs, T, "Review: Current International Research into Cellulose Nanofibres and Nanocomposites." J. Mater. Sci., 45 (1) 1-33 (2010)

2. Håkansson, KMO, Fall, AB, Lundell, F, Yu, S, Krywka, C, Roth, SV, Santoro, G, Kvick, M, Prahl Wittberg, L, Wågberg, L, Söderberg, LD, "Hydrodynamic Alignment and Assembly of Nanofibrils Resulting in Strong Cellulose Filaments." Nat. Commun., 54018 (2014)

3. Mittal, N, Jansson, R, Widhe, M, Benselfelt, T, Håkansson, KMO, Lundell, F, Hedhammar, M, Söderberg, LD, "Ultrastrong and Bioactive Nanostructured Bio-Based Composites." ACS Nano, 11 (5) 5148-5159 (2017)

4. Zhang, Y, Paris, O, Terrill, NJ, Gupta, HS, "Uncovering Three-Dimensional Gradients in Fibrillar Orientation in an Impact-Resistant Biological Armour." Sci. Rep., 6 (1) 26249 (2016)

5. Aulin, C, Gällstedt, M, Lindström, T, “Oxygen and Oil Barrier Properties of Microfibrillated Cellulose Films and Coatings." Cellulose, 17 (3) 559-574 (2010)

6. Li, Y, Yu, S, Chen, P, Rojas, R, Hajian, A, Berglund, L, "Cellulose Nanofibers Enable Paraffin Encapsulation and the Formation of Stable Thermal Regulation Nanocomposites." Nano Energy, 34 (March) 541-548 (2017)

7. Nogi, M, Iwamoto, S, Nakagaito, AN, Yano, H, "Optically Transparent Nanofiber Paper." Adv. Mater., 21 (16) 1595$1598(2009)$

8. Cervin, NT, Johansson, E, Larsson, PA, Wågberg, L, "Strong, Water-Durable, and Wet-Resilient Cellulose Nanofibril-Stabilized Foams from Oven Drying." ACS Appl. Mater. Interfaces., 8 (18) 11682-11689 (2016)

9. Köklükaya, O, Carosio, F, Grunlan, JC, Wågberg, L, "Flame-Retardant Paper from Wood Fibers Functionalized via Layer-by-Layer Assembly." ACS Appl. Mater. Interfaces., 7 (42) 23750-23759 (2015)

10. Innis-Samson, VA, Sakurai, K, "Swelling in Spin-Coated Methylcellulose Ultra-Thin Films: Effect on Film Structure, Surface Topography, and Temperature-Response Property." Soft Matter, 8 (28) 7351 (2012)

11. Ogihara, H, Xie, J, Okagaki, J, Saji, T, "Simple Method for Preparing Superhydrophobic Paper: Spray-Deposited Hydrophobic Silica Nanoparticle Coatings Exhibit High Water-Repellency and Transparency." Langmuir, 28 (10) 4605-4608 (2012)

12. Nash, C, Spiesschaert, Y, Amarandei, G, Stoeva, Z, Tomov, RI, Tonchev, D, van Driessche, I, Glowacki, BA, "A Comparative Study on the Conductive Properties of Coated and Printed Silver Layers on a Paper Substrate." J. Electron. Mater., 44 (1) 497-510 (2015)

13. Hamedi, MM, Hajian, A, Fall, AB, Håkansson, K, Salajkova, M, Lundell, F, Wågberg, L, Berglund, LA, "Highly Conducting, Strong Nanocomposites Based on NanocelluloseAssisted Aqueous Dispersions of Single-Wall Carbon Nanotubes." ACS Nano, 8 (3) 2467-2476 (2014)

14. Ngo, YH, Li, D, Simon, GP, Garnier, G, "Gold NanoparticlePaper as a Three-Dimensional Surface Enhanced Raman Scattering Substrate." Langmuir, 28 (23) 8782-8790 (2012)

15. Wei, H, Hossein Abtahi, SM, Vikesland, PJ, "Plasmonic Colorimetric and SERS Sensors for Environmental Analysis." Environ. Sci. Nano, 2 (2) 120-135 (2015)

16. Abraham, E, Weber, DE, Sharon, S, Lapidot, S, Shoseyov, $\mathrm{O}$, "Multifunctional Cellulosic Scaffolds from Modified Cellulose Nanocrystals." ACS Appl. Mater. Interfaces., 9 (3) 2010-2015 (2017)

17. Benselfelt, T, Pettersson, T, Wågberg, L, "Influence of Surface Charge Density and Morphology on the Formation of Polyelectrolyte Multilayers on Smooth Charged Cellulose Surfaces." Langmuir, 33 (4) 968-979 (2017)

18. Yoon, D, Kim, T, Moon, D, "Flexible Top Emission Organic Light-Emitting Devices Using Sputter-Deposited Ni Films on Copy Paper Substrates." Curr. Appl. Phys., 10 (4) e135e138 (2010) 
19. Yeh, T, Wang, Z, Mahajan, D, Hsiao, BS, Chu, B, "High Flux Ethanol Dehydration Using Nanofibrous Membranes Containing Graphene Oxide Barrier Layers." J. Mater. Chem. A, 1 (41) 12998 (2013)

20. Hu, L, Zheng, G, Yao, J, Liu, N, Weil, B, Eskilsson, M, Karabulut, E, Ruan, Z, Fan, S, Bloking, JT, McGehee, MD, Wågberg, L, Cui, Y, "Transparent and Conductive Paper from Nanocellulose Fibers." Energy Environ. Sci., 6 (2) $513-$ 518 (2013)

21. Roth, SV, Artus, GRJ, Rankl, M, Seeger, S, Burghammer, M, Riekel, C, Müller-Buschbaum, P, "Lateral Structural Variations in Thin Cellulose Layers Investigated by Microbeam Grazing Incidence Small-Angle X-Ray Scattering." Phys. B Condens. Matter, 357 (1-2 SPEC. ISS.) 190-192 (2005)

22. Blell, R, Lin, X, Lindström, T, Ankerfors, M, Pauly, M, Felix, O, Decher, G, "Generating in-Plane Orientational Order in Multilayer Films Prepared by Spray-Assisted Layer-by-Layer Assembly." ACS Nano, 11 (1) 84-94 (2017)

23. Zabihi, F, Eslamian, M, "Characteristics of Thin Films Fabricated by Spray Coating on Rough and Permeable Paper Substrates." J. Coat. Technol. Res., 12 (3) 489-503 (2015)

24. Seyrek, E.; Decher, G. Layer-by-Layer Assembly of Multifunctional Hybrid Materials and Nanoscale Devices. In Polymer Science: A Comprehensive Reference; Elsevier, 2012; Vol. 7, pp 159-185.

25. Shanmugam, K, Varanasi, S, Garnier, G, "Rapid Preparation of Smooth Nanocellulose Films Using Spray Coating." Cellulose, 24 (7) 2669-2676 (2017)

26. Schaaf, P, Voegel, J-C, Jierry, L, Boulmedais, F, "SprayAssisted Polyelectrolyte Multilayer Buildup: From Step-byStep to Single-Step Polyelectrolyte Film Constructions." Adv. Mater., 24 (8) 1001-1016 (2012)

27. Park, H-Y, Jin, J-S, Yim, S, Oh, S-H, Kang, P-H, Choi, S-K, Jang, S-Y, "Effects of Surface Characteristics of Dielectric Layers on Polymer Thin-Film Transistors Obtained by Spray Methods." Phys. Chem. Chem. Phys., 15 (11) 3718-3724 (2013)

28. Ummartyotin, S, Juntaro, J, Sain, M, Manuspiya, H, "Development of Transparent Bacterial Cellulose Nanocomposite Film as Substrate for Flexible Organic Light Emitting Diode (OLED) Display." Ind. Crops Prod., 35 (1) 92-97 (2012)

29. Velichko, EV, Buyanov, AL, Saprykina, NN, Chetverikov, YO, Duif, CP, Bouwman, WG, Smyslov, RY, "HighStrength Bacterial Cellulose-polyacrylamide Hydrogels: Mesostructure Anisotropy as Studied by Spin-Echo SmallAngle Neutron Scattering and Cryo-SEM." Eur. Polym. J., 88 269-279 (2017)

30. Schaub, M, Fakirov, C, Schmidt, A, Lieser, G, Wenz, G, Wegner, G, Albouy, PA, Wu, H, Foster, MD, Majrkzak, C, Satija, S, "Ultrathin Layers and Supramolecular Architecture of Isopentylcellulose." Macromolecules, 28 (4) 12211228 (1995)

31. Hexemer, A, Müller-Buschbaum, P, "Advanced GrazingIncidence Techniques for Modern Soft-Matter Materials Analysis." IUCrJ, 2 106-125 (2015)

32. Keckes, J, Burgert, I, Frühmann, K, Müller, M, Kölln, K, Hamilton, M, Burghammer, M, Roth, SV, Stanzl-Tschegg, S, Fratzl, P, "Cell-Wall Recovery after Irreversible Deformation of Wood." Nat. Mater., 2 (12) 810-813 (2003)

33. Kölln, K, Grotkopp, I, Burghammer, M, Roth, SV, Funari, SS, Dommach, M, Müller, M, "Mechanical Properties of Cellulose Fibres and Wood. Orientational Aspects in Situ Investigated with Synchrotron Radiation." J. Synchrotron Radiat., 12 (6) 739-744 (2005)
34. Mao, Y, Liu, K, Zhan, C, Geng, L, Chu, B, Hsiao, BS, "Characterization of Nanocellulose Using Small-Angle Neutron, X-Ray, and Dynamic Light Scattering Techniques." $J$. Phys. Chem. B, 121 (6) 1340-1351 (2017)

35. Ehmann, HMA, Werzer, O, Pachmajer, S, Mohan, T, Amenitsch, H, Resel, R, Kornherr, A, Stana-Kleinschek, K, Kontturi, E, Spirk, S, "Surface Sensitive Approach to Interpreting Supramolecular Rearrangements in Cellulose by Synchrotron Grazing Incidence Small Angle X-Ray Scattering." ACS Macro Lett., 4 (7) 1-10 (2015)

36. Daillant, J, Bélorgey, O, "Surface Scattering of X Rays in Thin Films. Part I. Theoretical Treatment." J. Chem. Phys., 97 (8) 5824 (1992)

37. Daillant, J, Gibaud, A, "X-Ray and Neutron Reflectivity." In: Daillant, J, Gibaud, A (eds.) Lecture Notes in Physics, Vol. 770. Springer, Berlin (2009)

38. Holy, V, Baumbach, T, "Introduction I. Nonspecular X-Ray Reflection from Rough Multilayers." Phys. Rev. B, 49 (15) 10668 (1994)

39. Schwartzkopf, M, Roth, S, "Investigating Polymer-Metal Interfaces by Grazing Incidence Small-Angle X-Ray Scattering from Gradients to Real-Time Studies." Nanomaterials, 6 (12) 239 (2016)

40. Levine, JR, Cohen, JB, Chung, YW, Georgopoulos, P, "Grazing-Incidence Small-Angle X-Ray Scattering: New Tool for Studying Thin Film Growth." J. Appl. Crystallogr., 22 (6) 528-532 (1989)

41. Yoneda, Y, "Anomalous Surface Reflection of X Rays." Phys. Rev., 131 (5) 2010-2013 (1963)

42. Dosch, H, Batterman, BW, Wack, DC, "Depth-Controlled Grazing-Incidence Diffraction of Synchrotron X Radiation." Phys. Rev. Lett., 56 (11) 1-4 (1986)

43. Jiang, Z, Lee, DR, Narayanan, S, Wang, J, Sinha, SK, "Waveguide-Enhanced Grazing-Incidence Small-Angle XRay Scattering of Buried Nanostructures in Thin Films." Phys. Rev. B, 84 (7) 75440 (2011)

44. Thompson, M, Sakamoto, R, Bernard, E, Kirby, N, Kluth, P, Riley, D, Corr, C, “GISAXS Modelling of Helium-Induced Nano-Bubble Formation in Tungsten and Comparison with TEM." J. Nucl. Mater., 473 6-12 (2016)

45. Lee, B, Park, I, Yoon, J, Park, S, Kim, J, Kim, K, Chang, T, Ree, M, "Structural Analysis of Block Copolymer Thin Films with Grazing Incidence Small-Angle X-Ray Scattering." Macromolecules, 38 (10) 4311-4323 (2005)

46. Hu, S, Rieger, J, Roth, SV, Gehrke, R, Leyrer, RJ, Men, Y, "GIUSAXS and AFM Studies on Surface Reconstruction of Latex Thin Films during Thermal Treatment $\uparrow$." Langmuir, 25 (7) 4230-4234 (2009)

47. Buffet, A, Rothkirch, A, Döhrmann, R, Körstgens, V, Abul Kashem, MM, Perlich, J, Herzog, G, Schwartzkopf, M, Gehrke, R, Müller-Buschbaum, P, Roth, SV, "P03, the Microfocus and Nanofocus X-Ray Scattering (MiNaXS) Beamline of the PETRA III Storage Ring: The Microfocus Endstation." J. Synchrotron Radiat., 19 (4) 647-653 (2012)

48. French, AD, "Idealized Powder Diffraction Patterns for Cellulose Polymorphs." Cellulose, 21 (2) 885-896 (2014)

49. Benecke, G, Wagermaier, W, Li, C, Schwartzkopf, M, Flucke, G, Hoerth, R, Zizak, I, Burghammer, M, Metwalli, E, Müller-Buschbaum, P, Trebbin, M, Förster, S, Paris, O, Roth, SV, Fratzl, P, "A Customizable Software for Fast Reduction and Analysis of Large X-Ray Scattering Data Sets: Applications of the New DPDAK Package to SmallAngle X-Ray Scattering and Grazing-Incidence Small-Angle X-Ray Scattering." J. Appl. Crystallogr., 47 (5) 1797-1803 (2014) 
50. Henriksson, M, Henriksson, G, Berglund, LA, Lindström, T, "An Environmentally Friendly Method for Enzyme-Assisted Preparation of Microfibrillated Cellulose (MFC) Nanofibers." Eur. Polym. J., 43 (8) 3434-3441 (2007)

51. Müller-Buschbaum, P, "Influence of Surface Cleaning on Dewetting of Thin Polystyrene Films." Eur. Phys. J. E Soft Matter, 12 (3) 443-448 (2003)

52. Song, L, Wang, W, Körstgens, V, Moseguí González, D, Löhrer, FC, Schaffer, CJ, Schlipf, J, Peters, K, Bein, T, Fattakhova-Rohlfing, D, Roth, SV, Müller-Buschbaum, P, "In Situ Study of Spray Deposited Titania Photoanodes for Scalable Fabrication of Solid-State Dye-Sensitized Solar Cells." Nano Energy, 40 (August) 317-326 (2017)

53. Al-Hussein, M, Herzig, EM, Schindler, M, Löhrer, F, Palumbiny, CM, Wang, W, Roth, SV, Müller-Buschbaum, $\mathrm{P}$, "Comparative Study of the Nanomorphology of Spray and Spin Coated PTB7 Polymer: Fullerene Films." Polym. Eng. Sci., 56 (8) 889-894 (2016)

54. Al-Hussein, M, Schindler, M, Ruderer, MA, Perlich, J, Schwartzkopf, M, Herzog, G, Heidmann, B, Buffet, A, Roth, SV, Müller-Buschbaum, P, "In Situ X-Ray Study of the Structural Evolution of Gold Nano-Domains by Spray Deposition on Thin Conductive P3HT Films." Langmuir, 29 (8) 2490-2497 (2013)

55. Herzog, G, Benecke, G, Buffet, A, Heidmann, B, Perlich, J, Risch, JFH, Santoro, G, Schwartzkopf, M, Yu, S, Wurth, W, Roth, SV, "In Situ Grazing Incidence Small-Angle X-Ray Scattering Investigation of Polystyrene Nanoparticle Spray Deposition onto Silicon." Langmuir, 29 (36) 11260-11266 (2013)

56. Zhang, P, Santoro, G, Yu, S, Vayalil, SK, Bommel, S, Roth, SV, "Manipulating the Assembly of Spray-Deposited
Nanocolloids. In Situ Study and Monolayer Film Preparation.” Langmuir, 32 (17) 4251-4258 (2016)

57. Roth, SV, "A Deep Look into the Spray Coating Process in Real-Time-the Crucial Role of X-Rays." J. Phys.: Condens. Matter, 28 (40) 403003 (2016)

58. Jiang, Z, "GIXSGUI: A MATLAB Toolbox for GrazingIncidence X-Ray Scattering Data Visualization and Reduction, and Indexing of Buried Three-Dimensional Periodic Nanostructured Films." J. Appl. Crystallogr., 48 917-926 (2015)

59. Nishiyama, Y, Langan, P, Chanzy, H, "Crystal Structure and Hydrogen-Bonding System in Cellulose I $\beta$ from Synchrotron X-Ray and Neutron Fiber Diffraction." J. Am. Chem. Soc. , 124 (31) 9074-9082 (2002)

60. Perlich, J, Kaune, G, Memesa, M, Gutmann, JS, MullerBuschbaum, P, "Sponge-like Structures for Application in Photovoltaics." Philos. Trans. R. Soc. A Math. Phys. Eng. Sci., 2009 (367) 1783-1798 (1894)

61. Schaffer, CJ, Palumbiny, CM, Niedermeier, MA, Jendrzejewski, C, Santoro, G, Roth, SV, Müller-Buschbaum, P, “A Direct Evidence of Morphological Degradation on a Nanometer Scale in Polymer Solar Cells." Adv. Mater., 25 (46) 6760-6764 (2013)

62. Hosemann, R, Bagchi, SN, "The Interference Theory of Ideal Paracrystals." Acta Crystallogr., 5 (5) 612-614 (1952)

63. Müller-Buschbaum, P, "Grazing Incidence Small-Angle XRay Scattering: An Advanced Scattering Technique for the Investigation of Nanostructured Polymer Films.' Anal. Bioanal. Chem., 376 (1) 3-10 (2003)

64. Renaud, G, Lazzari, R, Leroy, F, "Probing Surface and Interface Morphology with Grazing Incidence Small Angle X-Ray Scattering." Surf. Sci. Rep., 64 (8) 255-380 (2009) 